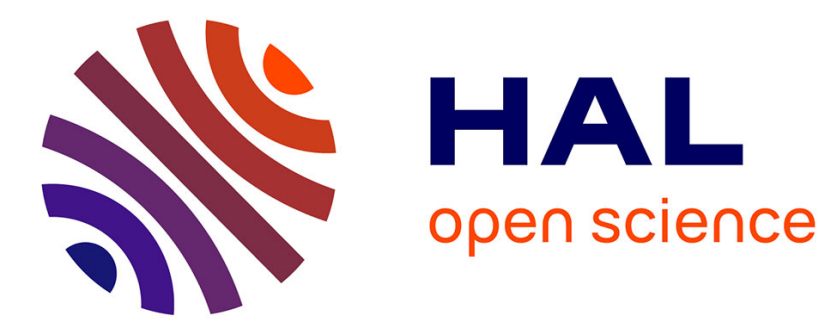

\title{
L'atmosphère de la Terre s'étend bien au-delà de la Lune Jean-Loup Bertaux
}

\section{To cite this version:}

Jean-Loup Bertaux. L'atmosphère de la Terre s'étend bien au-delà de la Lune. La Météorologie, 2019, $\mathrm{N}^{\circ} 105$ (Mai), pp.4-5. insu-02332533

\section{HAL Id: insu-02332533 \\ https://hal-insu.archives-ouvertes.fr/insu-02332533}

Submitted on 4 Jun 2021

HAL is a multi-disciplinary open access archive for the deposit and dissemination of scientific research documents, whether they are published or not. The documents may come from teaching and research institutions in France or abroad, or from public or private research centers.
L'archive ouverte pluridisciplinaire HAL, est destinée au dépôt et à la diffusion de documents scientifiques de niveau recherche, publiés ou non, émanant des établissements d'enseignement et de recherche français ou étrangers, des laboratoires publics ou privés. 


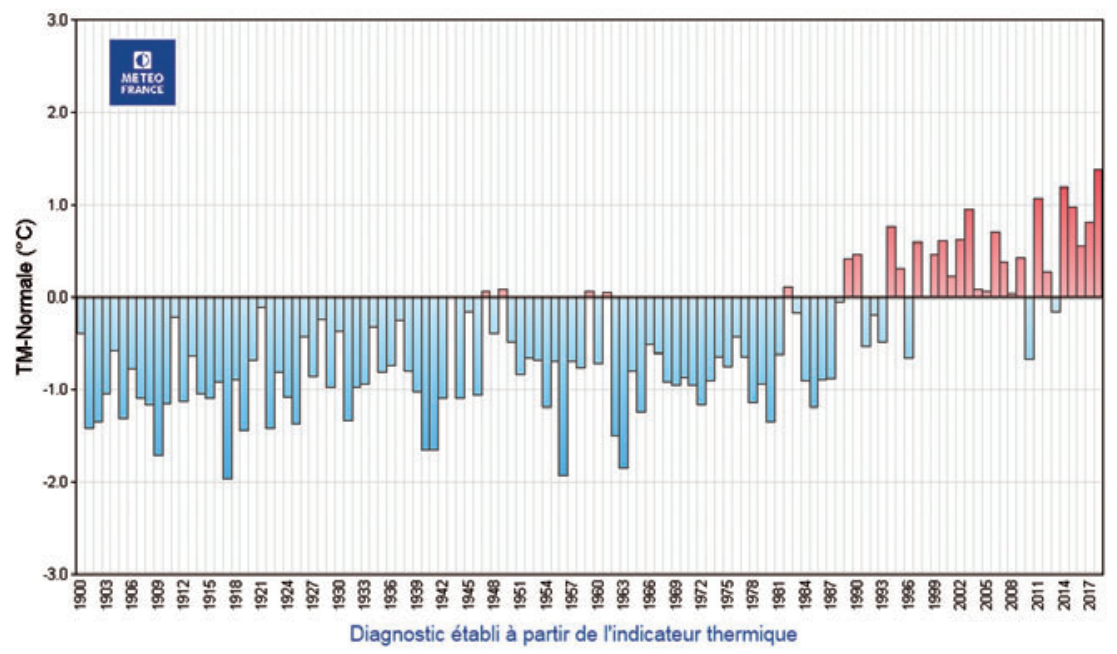

Figure 4. Écart à la moyenne annuelle de référence 1981-2010 de l'indicateur de température moyenne en France. Source : Météo-France.

dehors de février et mars, tous les mois ont des températures supérieures aux normales. C'est la première fois depuis le début des mesures que l'on note une séquence de 9 mois consécutifs au- dessus des normales. Janvier est un mois particulièrement remarquable, avec une moyenne de $8,4{ }^{\circ} \mathrm{C}$ (excédent de $3,4{ }^{\circ} \mathrm{C}$ ) ; février contraste vigoureusement avec le mois précédent (moyenne de seulement $3,5^{\circ} \mathrm{C}$, déficit de $2,2{ }^{\circ} \mathrm{C}$ ). Durant l'année, la France a connu des inondations et des tempêtes en janvier, une canicule du 24 juillet au 8 août, une sécheresse d'août à novembre dans plusieurs régions et des pluies générant des crues dévastatrices et meurtrières dans le Midi, notamment l'Aude les 14 et 15 octobre (15 morts et 220 millions d'euros de dégâts).

Selon Météorage, l'année 2018 a été l'année la plus orageuse en France depuis le début des mesures il y a près de 30 ans, avec 724953 éclairs nuage/sol (maximum mensuel en mai : 182 127) ; le 9 août, jour de la Saint-Amour, a été la journée la plus orageuse avec 41639 coups de foudre... La commune la plus foudroyée a été La Favière (Jura).

\section{Guy Blanchet}

Directeur honoraire du centre de climatologie de l'université Claude-Bernard, Lyon 1

\section{Sources}

http://www.ncdc.noaa.gov/sotc/global/20113

https://data.giss.nasa.gov/gistep/maps

http://www.meteofrance.fr/climat-passe-et-futur/bilans-climatiques/bilan-2018/bilan-climatique-de-1-annee-2018

https://www.ncdc.noaa.gov/cag/global/time-series

http://www.meteofrance.fr/actualites/70734051-2018-4e-annee-la-plus-chaude-sur-le-globe

https://public.wmo.int/fr

https://nsidc.org/arcticseaicenews/

\section{L'atmosphère de la Terre s'étend bien au-delà de la Lune}

"Dans l'orbite de la Terre

Quand la planète n'est plus

Au loin qu'une faible sphère,

Qu'entoure un rêve ténu... "

Quand le poète Jules Supervielle ${ }^{1}$ écrivit ces vers en 1925, il n'imaginait pas que l'atmosphère de la Terre, ce rêve ténu, s'étendait bien au-delà de l'orbite de la Lune. Et les astronautes des missions Apollo, debout sur la Lune, ne se doutaient pas qu'ils baignaient dans cette atmosphère et qu'ils recevaient, en regardant au loin la faible sphère, un vent soufflant à Mach 3 sur leur scaphandre!

C'est pourtant ce que vient de démontrer une étude récente (Baliukin et al., 2019) en analysant des observations réalisées depuis le satellite Soho ${ }^{2}$ en 1996, 1997 et 1998 par l'instrument Swan ${ }^{3}$. Cet instrument franco-finlandais est capable de dresser une carte complète du ciel en 24 heures en lumière ultraviolette (UV), incluant l'émission Lyman-alpha ( $\mathrm{L} \alpha)$ spécifique de l'atome d'hydrogène à la longueur d'onde de 121,6 nm, impossible à voir depuis le sol, car la basse atmosphère est opaque à cette longueur d'onde. C'est l'équivalent pour l'atome d'hydrogène de la couleur jauneorange spécifique de l'atome de sodium, bien connue car utilisée dans les lampes à sodium pour l'éclairage urbain. Le Soleil est une source très intense de cette lumière $\mathrm{L} \alpha$ qui éclaire tous les atomes d'hydrogène présents dans le système solaire. Ceux-ci diffusent alors par résonance optique les photons solaires. Des atomes d'hydrogène en provenance du milieu interstellaire traversent en permanence le système solaire, mais sont détruits par le vent solaire. On conçoit donc que la distribution de lumière $\mathrm{L} \alpha$ sur le ciel dépend de la distribution $\mathrm{du}$ vent solaire en fonction de la latitude solaire, et l'objectif de Swan était effectivement de mesurer ces anisotropies du vent solaire en relevant des cartes UV du ciel tous les deux jours et de déterminer ses variations au cours du cycle solaire de 11 ans. Ce qui fut fait et continue de nos jours.
Mais depuis son poste d'observation au point de Lagrange L1 (Soho est à 1,5 million de kilomètres de la Terre en direction du Soleil), Swan a également cartographié (figure 1) l'émission des atomes d'hydrogène de l'atmosphère terrestre et constaté que cette émission est détectable jusqu'à $630000 \mathrm{~km}$ de la Terre, alors que l'orbite de la Lune est à $384000 \mathrm{~km}$ (figure 2) !

Pour identifier la limite de l'atmosphère, il fallait distinguer l'hydrogène originaire de l'atmosphère de celui provenant du milieu interstellaire. Pour cela, l'instrument Swan possède une cellule à absorption d'hydrogène capable de différencier les émissions des deux sources par effet Doppler (en gros, la cellule absorbe l'émission terrestre, mais n'absorbe pas

1. Première strophe du poème Souffle de Jules Supervielle, paru en 1925 dans le recueil Gravitations.

2. SOlar Heliospheric Observatory, un programme ESA/Nasa.

3. Solar Wind ANisotropies. 


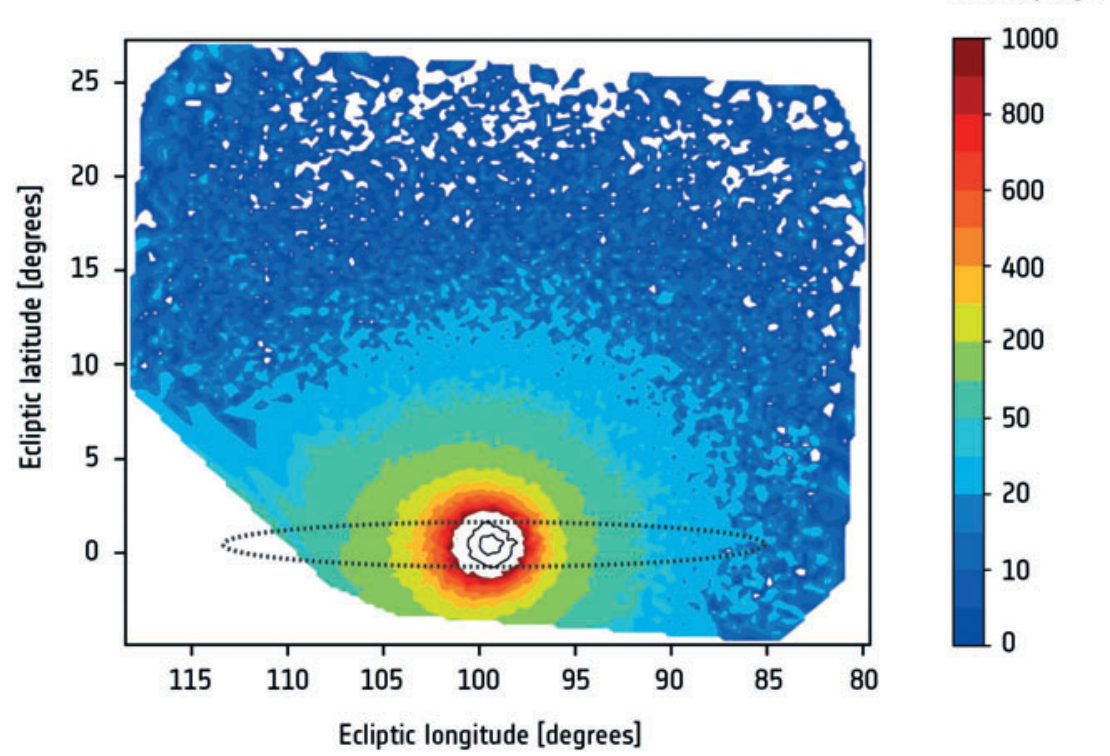

Figure 1. Carte de l'intensité lumineuse de la géocouronne en lumière monochromatique à Lyman-alpha $=121,6 \mathrm{~nm}$, codée en fausses couleurs en unités Rayleigh. L'orbite de la Lune de $384000 \mathrm{~km}$, centrée sur la Terre, est représentée en pointillés et donne l'échelle du phénomène. Cette carte a été relevée en 1997 depuis le satellite Soho, un projet ESA/Nasa d'observatoire du Soleil et de l'héliosphère.

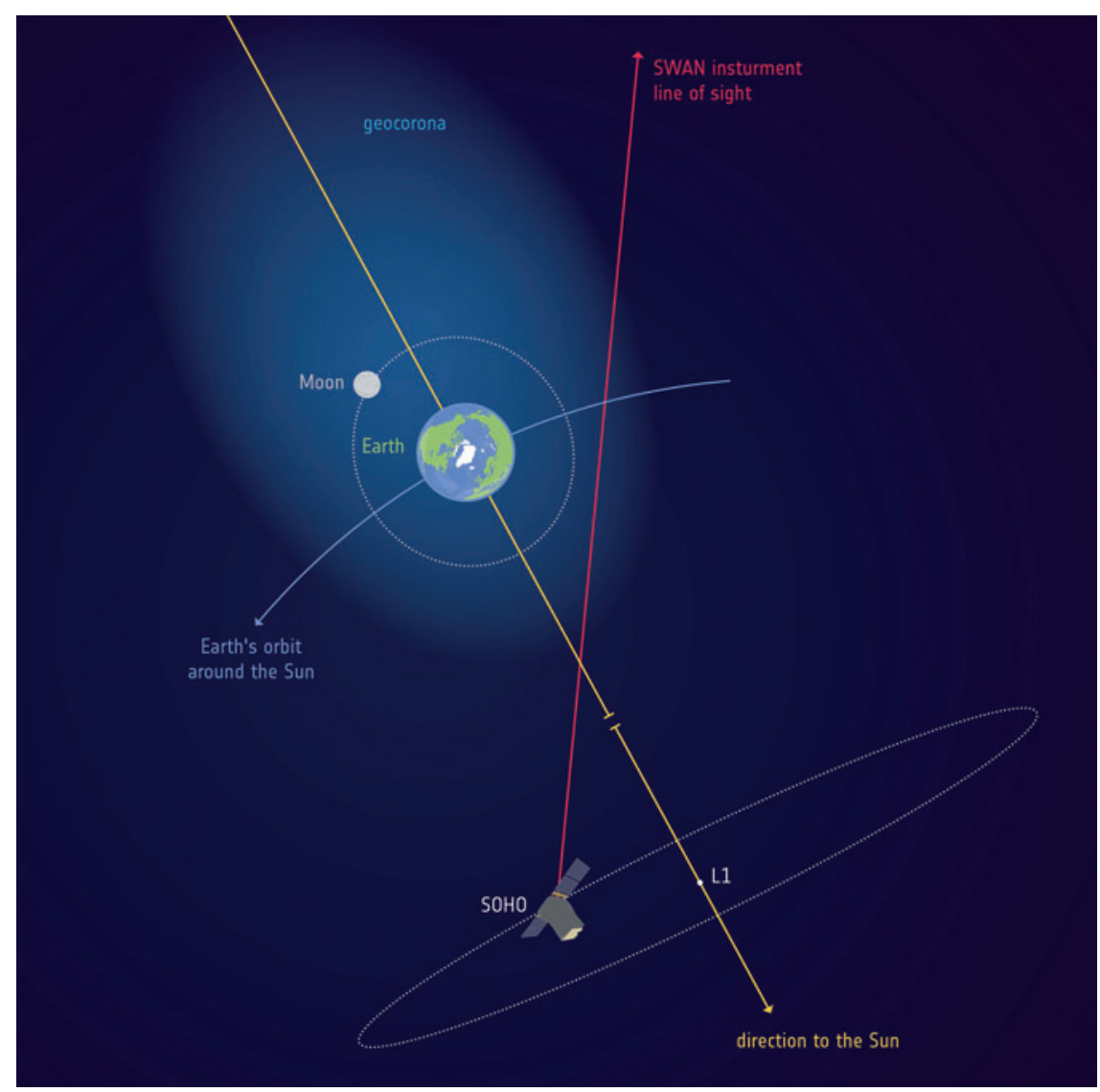

Figure 2. Configuration géométrique des observations de Swan à bord du satellite Soho, tournant autour du point de Lagrange L1. La dimension de la Terre est fortement exagérée. La géocouronne est allongée dans la direction anti-solaire par la pression de radiation du rayonnement $L \alpha$ solaire.

l'émission interplanétaire à cause de la vitesse orbitale de la Terre dans le système solaire).

Les atomes d'hydrogène que l'on voit proviennent de la photodissociation de la vapeur d'eau vers 80-100 km d'altitude. Puis, ces atomes, les plus légers, diffusent à travers la thermosphère $(100-500 \mathrm{~km})$ pour atteindre l'exosphère à $500 \mathrm{~km}$, définie comme un milieu sans collision, car peu dense. À cette altitude, chaque atome d'hydrogène suit alors une trajectoire balistique. Si sa vitesse est inférieure à la vitesse de libération $(11,2 \mathrm{~km} / \mathrm{s})$, il retombe dans l'atmosphère, à $500 \mathrm{~km}$ d'altitude. Si sa vitesse est supérieure à $11,2 \mathrm{~km} / \mathrm{s}$, sa trajectoire est une hyperbole qui l'entraîne vers l'infini. Soit une perte sèche pour la Terre correspondant, au taux actuel d'échappement, à environ 1 mètre d'eau liquide par milliard d'années. On peut dire que l'exosphère de la Terre est constituée uniquement d'hydrogène atomique; et depuis que l'astrophysicien ukrainien et soviétique Iossif Shklovsky a proposé en 1959 le mot géocouronne pour désigner une possible composante d'hydrogène dans l'atmosphère, ce mot désigne non seulement l'exosphère, mais aussi l'hydrogène compris entre 100 et $500 \mathrm{~km}$ d'altitude.

La géocouronne fut, au début de l'ère spatiale, un objet d'étude privilégié. Notamment grâce à Jacques Blamont, directeur du Service d'aéronomie du CNRS, avec son expérience E-22 sur le satellite $O G O-5$ (1968-1971) de la Nasa qu'on peut considérer comme un précurseur de Swan (Bertaux, 1974), dédié à la géocouronne, ainsi que le satellite français $D-2 A$ Tournesol du Cnes, voguant en 1971 autour de la base de l'exosphère vers $500 \mathrm{~km}$ d'altitude.

Naturellement, la densité de l'atmosphère décroît quand l'altitude croît. Au sol, la densité est de 2,7 × $10^{19}$ molécules par $\mathrm{cm}^{3}$, mais il n'y a plus que $10^{5}$ atomes d'hydrogène par $\mathrm{cm}^{3}$ à $500 \mathrm{~km}$ d'altitude, puis 70 atomes $/ \mathrm{cm}^{3}$ à $60000 \mathrm{~km}$, seulement 0,2 atome $/ \mathrm{cm}^{3}$ au niveau de la Lune et 0,05 atome $/ \mathrm{cm}^{3}$ à $637000 \mathrm{~km}$. Autant dire le vide... mais pas tout à fait, puisqu'on peut encore $\mathrm{y}$ détecter ces atomes d'hydrogène éclairés par le Soleil. Avant Swan, les astronautes d'Apollo 16 avaient mis en œuvre une caméra UV sur le sol de la Lune (1972), permettant une prise de vue magnifique de la Terre et de sa géocouronne (figure 3) et la détectant jusqu'à $103000 \mathrm{~km}$. Plus récemment, un microsatellite japonais prenait en 2015 un cliché UV depuis une distance de 15 millions de kilomètres, révélant une extension de la géocouronne jusqu'à environ $300000 \mathrm{~km}$. Swan double ainsi la taille de cette géocouronne, qui englobe désormais l'orbite lunaire et au-delà. Cela est insuffisant pour freiner la Lune, qui s'éloigne de la Terre de $3 \mathrm{~cm}$ par an. Par contre, le Soleil exerce une pression de radiation sur les atomes d'hydrogène, ce qui se traduit par une forme un peu allongée de l'exosphère dans la direction anti-solaire, baptisée geotail 
par les chercheurs impliqués dans $O G O-5$ qui en firent la première observation en 1971.

Une conséquence de la grande extension de la géocouronne est que les télescopes spatiaux qui seront déployés en orbite autour de la Lune, ou sur son sol, verront cette émission $L \alpha$ de l'hydrogène, où qu'ils regardent. Cela est déjà le cas du Hubble Space Telescope gravitant à $500 \mathrm{~km}$. Cette "pollution » de la géocouronne est connue et il faut en tenir compte dans l'analyse de toutes les observations sensibles à la longueur d'onde $\mathrm{L} \alpha$.

Dans le cadre de l'exploration des planètes extrasolaires, l'existence

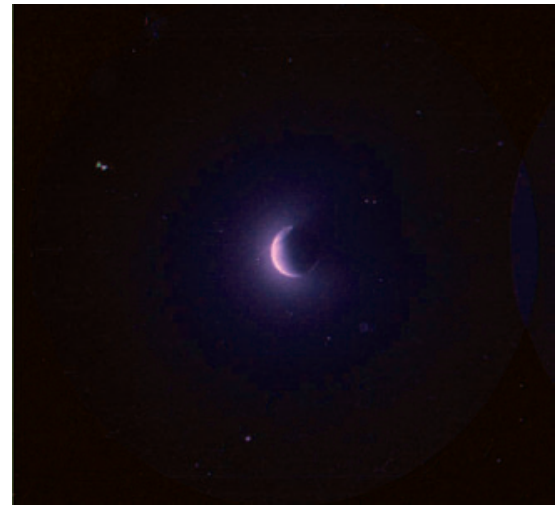

Figure 3. Cliché dans l'ultraviolet pris depuis le so lunaire au cours de la mission Apollo 16 en avril 1972 avec la caméra de Georges Carruthers. On distingue bien le globe terrestre et la géocouronne interne, mais le champ de vue est limité à une dizaine de rayons terrestres. d'exosphères étendues autour des trois planètes telluriques que sont Vénus, la Terre et Mars donne un regain d'intérêt à leur étude : peut-on détecter des exosphères d'hydrogène autour d'exoplanètes habitables de type terrestre ? Sans doute, mais bien que ces atomes d'hydrogène révèlent chez nous la présence d'eau dans l'atmosphère de nos trois planètes, il suffit de bien peu d'eau pour avoir une exosphère, comme nous le montre Vénus, qui n'a que $3 \mathrm{~mm}$ équivalent liquide sous forme de vapeur : attention donc aux faux positifs !

Jean-Loup Bertaux Laboratoire atmosphères, milieux, observations spatiales, CNRS/UVSQ Space Research Institute de l'Académie des sciences de Russie, Moscou, Russie

Baliukin I.I., Bertaux J.-L., Quémerais E., Izmodenov V.V., Schmidt W., 2019. SWAN/SOHO Lyman-alpha mapping: The hydrogen geocorona extends well beyond the Moon. Journal of Geophysical Research: Space Physics, 124, 861-885. doi:10.1029/2018JA026136

Bertaux J.-L., 1974. L'hydrogène atomique dans l'exosphère terrestre : mesures d'intensité et de largeur de raie de l'émission Lyman-alpha à bord du satellite OGO-5 et interprétation. Thèse de Doctorat d’État, sous la direction de J. Blamont, Université Paris 6.

\section{La participation des scientifiques français à CMIP6}

Les climatologues travaillant à l'Institut Pierre-Simon Laplace (IPSL), au Centre national des recherches météorologiques (CNRM, MétéoFrance/CNRS) et au laboratoire " Climat, environnement, couplages et incertitudes » (Cerfacs/ CNRS) ont annoncé la publication de nouveaux jeux de données qui promettent d'apporter de nouvelles perspectives sur les changements climatiques passés et futurs. Ces laboratoires sont en effet engagés dans un effort international pour fournir une nouvelle génération d'expériences numériques sur le changement climatique. Cela a nécessité le développement d'une nouvelle génération de modèles de climat. Ceux-ci comportent de nombreuses améliorations dans la représentation du système climatique de la Terre ainsi qu'une meilleure résolution spatiale. Les jeux de données, hébergés à l'IPSL et à Météo-France, et recopiés sur d'autres sites à travers le monde, sont mis gratuitement à la disposition de tous et permettront à une large communauté internationale de chercheurs d'analyser et d'examiner les résultats. Les principaux objectifs sont de comprendre les processus qui sous-tendent le changement climatique, d'évaluer les forces et les limites des modèles de climat et de fournir une base scientifique solide pour les politiques d'adaptation et d'atténuation au changement climatique.
La publication de ces ensembles de données est le résultat de plus de trois années de travail impliquant une

centaine de scientifiques investis dans l'infrastructure de recherche Climeri-France. En effet, la

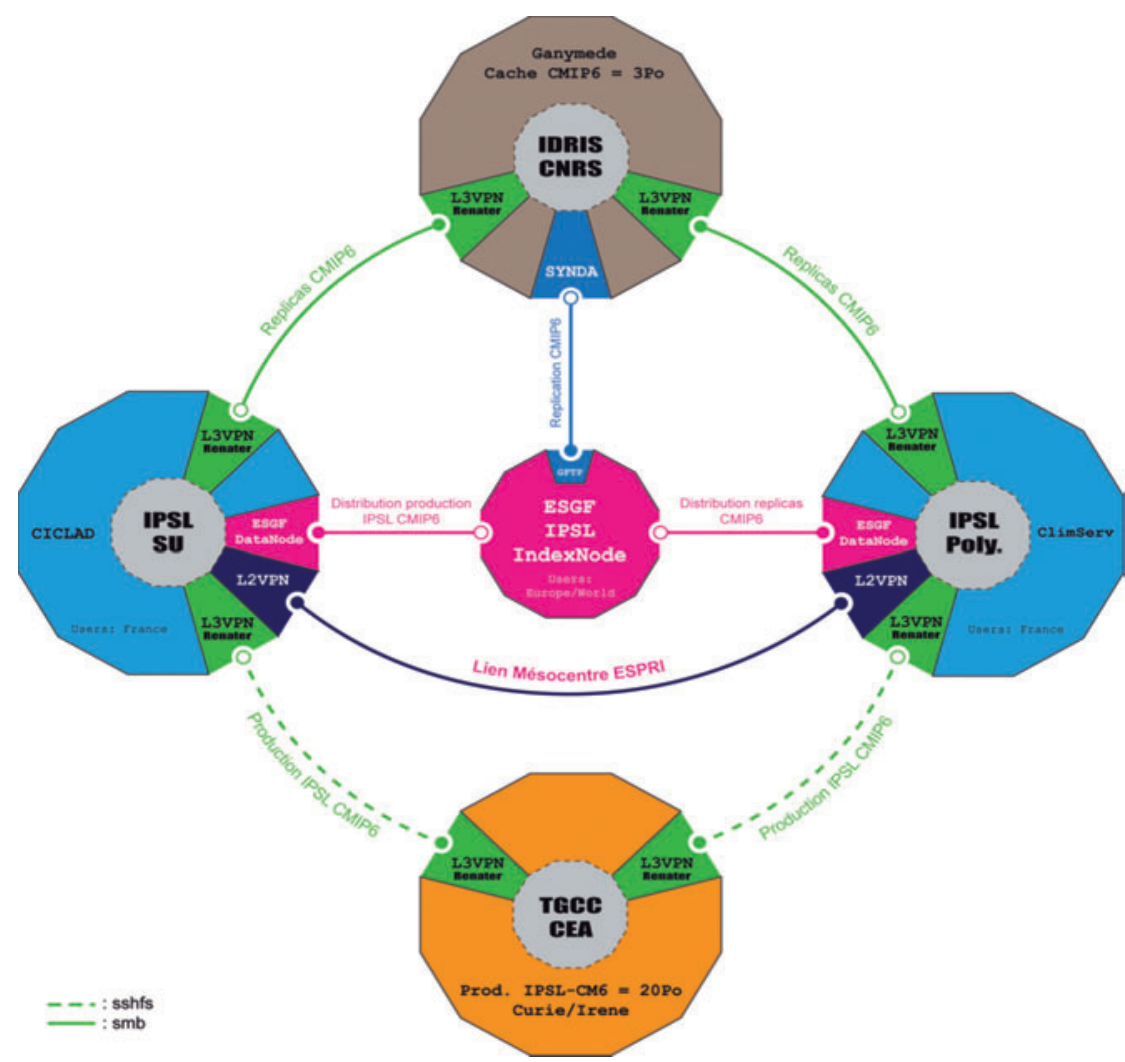

Figure 1. Architecture de l'infrastructure de calcul et de distribution des données servant au Centre de modélisation du climat de I'IPSL. Le mésocentre de calcul de I'IPSL permettant d'analyser les données climatiques est relié aux centres de calcul nationaux Idris-CNRS et TGCC-CEA. Le nœud de distribution ESGF s'appuie également sur le stockage de masse positionné dans les deux centres de calcul. (c) Guillaume Levavasseur, Sorbonne Université 